\title{
Definition and detection of simulation noise via imaginary simulated particles in comparison with an electrical microfluidic chip noise
}

\author{
M. Tahsin Guler ${ }^{1,2}$ \\ Received: 19 July 2020 / Accepted: 19 October 2020 \\ (C) Springer-Verlag GmbH Germany, part of Springer Nature 2020
}

\begin{abstract}
Real problems in science and engineering generally do not have an analytical solution, which invariably leads to the application of numerical methods to analyze the problem. The numerical solutions to the same problem give different results due to variations in discretization, which are defined as simulation noise in this study. Microfluidics impedance flow cytometry is employed to demonstrate and compare experimental and simulated noise. For measurement of the simulation noise, an object is assigned with the same electrical parameters as the medium and moved along the electrode region through a microchannel. Since the object is no different to the medium in terms of material properties, forwarding of the object through the electrodes doesn't have any physical effect, but just reorders the meshing. However, the impedance, which is the calculated output parameter of the simulation, fluctuates due to the reordering of the meshes and is defined as the simulation noise. By employing the imaginary object method, noise can be measured for every Finite element method (FEM) simulation even if the problem has a different physical background.
\end{abstract}

\section{Introduction}

Modeling a real-world problem or designing a device is one of the most important parts of science and engineering. Before fabricating a device, some simulations are performed to determine the design parameters, such as the dimensions, the materials to be used, etc. In order to discover the optimum parameters, many experimental processes need to be repeated several times, which increases the cost. Simulation comes into play at this point to save a lot of time and effort that also minimizes the costs. Hence developers generally rely on simulation results during the design stages of a device.

Many phenomena in the real-world can be defined by a partial differential equation (PDE). As the

Electronic supplementary material The online version of this article (https://doi.org/10.1007/s00542-020-05078-z) contains supplementary material, which is available to authorized users.

M. Tahsin Guler

gulermt@gmail.com

1 Department of Physics, Faculty of Arts and Science, Kirikkale University, 71450 Kirikkale, Turkey

2 UNAM, Institute of Materials Science and Nanotechnology, Bilkent University, 06800 Ankara, Turkey approximation to the problem becomes more realistic, the analytical solution becomes harder to determine. Hence, there is no analytical solution for many problems, especially once a fully realistic approximation is employed. The finite element method (FEM) is one of the numerical methods applied to solve a PDE that represents the physical situation. Many types of realistic problems that are defined by a PDE can only be analyzed with numerical methods, like problems in electromagnetism (Bilican et al. 2016), fluid mechanics (Su et al. 2019), Micro-Electro-Mechanical-Systems (MEMS) (Kumar et al. 2019; Thalluri et al. 2020), acoustics (Rahnama 2020), optics (Hah 2018), solid mechanics (Wang et al. 2018; Azeman et al. 2019), and heat transfer (Dhara and Singh 2020). The analytical solution becomes impossible to determine when all the effects of the problem are considered, and that makes a numerical solution the sole method to be employed.

Use of numerical methods to solve a PDE is spreading very fast thanks to user-friendly simulation programs and rising computational power. The FEM is one of the most commonly adopted methods among all simulation programs. A search in Google Scholar with the term 'Finite Element Method' gives nearly 4 million results, 119,000 of which come only from the year of 2019, demonstrating the trending aspect of the method. 
FEM offers a simple way, e.g. like approaching the problem of finding the perimeter of a circle by finding the perimeter of a polygon fitting inside or outside the circle. Because the straight line segments of a polygon can be measured one by one, the method does not require the analytical formula for the perimeter of a circle, which is $\mathrm{P}=2 \pi \mathrm{r}$. However, an error comes up in finding the exact perimeter, which depends on the discretization. When the discretization of the circle approaches infinity, the error goes to zero, but the calculation of an infinite number of pieces is impossible. Hence, an error is present in numerical solutions based on the discretization of the geometry.

Three types of error exist in FEM that arise from the approximation of the geometrical problem domain, the approximation of the analytical solution and the numerical error arising from the calculations (Reddy 1993). There are some methods that aim to improve the errors in FEM like X-FEM (Swati et al. 2019), Smoothed FEM (Vo-Duy et al. 2018), mesh adaptation (Wise et al. 2018), moving mesh (Gao and Zhang 2019), control volume (Saad et al. 2018), mixed FEM (Banz et al. 2019) etc. Separately, some meshfree methods have been developed to remove the meshing error that includes other error sources that comes into play (Abidou et al. 2017). In this report, COMSOL is employed as the simulation software that uses the FEM method to solve the related PDE. However, the philosophy behind this work should not be restricted to FEM, since the other numerical methods (Ames 2014) include similar perspectives.

The phrase 'meshing noise' was first used by Axelrad et al. (1997), Axelrad (1998) in 1997 for a statistical Technology Computer-Aided Design (TCAD) investigation of a CMOS device. Shigyo et al. published a study considering the meshing noise problem in the simulation of a MOSFET device (Shigyo et al. 2000), which was followed by Caire and Chifflet work (2002). Axelrad's work investigates the effect of mesh density on accuracy by assuming that the calculations which are done using denser mesh are more accurate and offers a different meshing algorithm having a relatively lower density that gives closer results to high-density meshing. Shigyo et al. showed that some physically irrelevant results are achieved with lower density meshing that can be fixed by using higher density mesh distribution. Caire et al. showed effect of the meshing on the results by changing the geometrical positions of some objects inside the simulated device that leads to altered result. Yet, as the variation of the simulation output can also arise from the shift at the geometry, the variance at the results can not be directly attributed to the meshing alone. Though being the first studies using the term meshing noise, these studies didn't receive much attention due to their method of handling the problem, as the phenomenon was considered to be a standard simulation convergence problem. Another drawback of these studies was the lack of experimental work to compare with the numerical results. Whereas in this work, the meshing noise is shown in several mesh densities with a method that just induce mesh reorientation as well as comparing with the experimental results.

This study will be the first time in literature that the error in the output of a simulation has been treated as noise, like it is in the experiment. Errors in a simulation arise from several facts including numerical errors during calculation as well as discretization errors during mesh construction. Here, only the discretization error is investigated and defined as simulation noise. In addition, a method for measuring the simulation noise was introduced, and the simulation noise was compared with the experimental noise for several parameters. Since the simulated device was a sensor, the comparison of the simulation noise with the experimental noise was done directly. Noise in the electrical circuit is the fluctuations of the current on an electrical element. The phenomenon has both extrinsic and intrinsic origins. Coupling of an electromagnetic wave to the circuit or big distortions induced by high power systems can be counted as extrinsic sources of noise. The spontaneous movement of the charge carriers due to thermal energy form the intrinsic noise that is called thermal noise or Johnson-Nyquist noise, which has a constant spectral density (Johnson 1928). There is also another kind of noise showing spectral variable power density like flicker noise, which has frequency dependence as 1/f over the entire spectrum (Handel 1993). The stochastic microscopic motion of the charge carriers forms voltage fluctuation on a resistor according to the Ohm's law that generates the noise in the electronic circuit. The noise is present not only in the electronic circuits, it is also present everywhere including stock markets, brain, and music notes in terms of flicker noise (Gilden et al. 1995; Voss and Clarke 1975). Besides, all the objects have thermal vibration although they normally look stabile. The exact origin and spectral properties of the noise are still unclear and debates are going on the topic.

The experiments and the simulations were carried out on an electrical microfluidic device. The microfluidic chip, which included an integrated micro-electrical module, was fabricated in a cleanroom employing a standard photolithography technique. The fabricated device connected to a lock in amplifier to detect the microbeads that were sent through the microfluidics chip, as shown before (Serhatlioglu et al. 2019; Errico et al. 2017; Caselli et al. 2018). In the simulation part, a parametric sweep was performed with COMSOL to simulate the particle moving in the microchannel. The parametric sweep was run twice; the first sweep was done after assigning the correct electrical permittivity and the resistivity parameters to the 
spherical object that represented the microbeads. The second sweep was performed after assigning the same electrical parameters to the spherical object in the solution that is called a imaginary particle namely the ghost particle. The current flowing through the electrodes was calculated for every step in each case. The first sweep gave the signal coming from the particle, while the second sweep produced only the noise of the simulation, and they were then compared with the experimental results.

\section{Materials and methods}

Fabrication of the device was done in a class 100 and class 1000 cleanroom in the UNAM (National Nanotechnology Research Center) facility of the Bilkent University. Production of the microfluidics device included three steps, which were the fabrication of the microelectrodes, the fabrication of the microchannel and the bonding of both parts. Finally, the device was connected to the measurement setup, followed by installation of the tubing to the inlet of the microfluidic chip.

\subsection{Microelectrode fabrication}

Microelectrode fabrication used the standard lift-off process on a glass slide. The fabrication process starts with the classical cleaning procedure. After cleaning, the substrate is spin-coated with HMDS and AZ5214E (Microchemicals) photoresist (PR), which is then followed by UV exposure. The UV patterned glass substrate is hung inside the thermal evaporator chamber and then coated with $10 \mathrm{~nm}$ chromium and $60 \mathrm{~nm}$ gold, respectively. The metalized substrate is dipped into acetone that etches the remaining PR so that peeling off the metal layer that was coated right over the PR finishes the microelectrode fabrication process. The schematic representation of the microelectrode fabrication is shown in Fig. 1 whose detail can be found on previous work (Bilican et al. 2016). In this study, electrodes are fabricated with width dimensions of $10 \mu \mathrm{m}$ and a gap that enables less signal variation according to the particle height from the channel base.

\subsection{Microchannel fabrication}

The microchannels are produced using a soft lithography technique. Initially, the mold is fabricated by taking a PDMS replica (Polydimethylsiloxane, Sylgard 184) to form the microchannels. A $500 \mu \mathrm{m}$-thick silicon wafer is used as the substrate for the mold fabrication. The wafer is only nitrogen cleaned. Then SU8 2008 photoresist is spincoated on the surface at $1000 \mathrm{rpm}$, which is followed by the baking and UV exposure steps. No mask is used for the initial UV exposure since the first spin-coated SU8 layer needs to remain on the surface as an adhesion layer between the following spin-coated SU8 layer and the wafer. The second layer of the SU8 2005 is spin-coated over the first layer at $1000 \mathrm{rpm}$. UV exposure is done with the mask aligner using a previously designed and printed soda lime mask. Then, the wafer is dipped into developer (mr-Dev 600, Micro Resist Technology); the structure formation on the surface is checked a few times by the naked eye and with a microscope each time. The substrate and the SU8 layers are fixed with an epoxy resin that is brushed onto the edge of the wafer. A gluing process is applied to prevent peeling of the SU8 from the wafer during PDMS replication. After waiting $8 \mathrm{~h}$, the mold fabrication process is finished and then followed by PDMS replication.

PDMS is mixed in a ratio of 1:10 (w/w) with the curing agent, and the mixture is vigorously stirred for better blending. The air bubbles formed inside the mixture are eliminated by degassing in a vacuum chamber. The mixture is poured onto the mold slowly and baked at $100{ }^{\circ} \mathrm{C}$ for $3 \mathrm{~h}$. After hardening, the PDMS is peeled off from the mold, which is followed by punching an inlet and outlet hole on the PDMS microchannel. Then, the PDMS is put on the glass slide with the microelectrodes on the surface. Both parts are sandwiched between the two laser-cut Plexiglas parts finishing the fabrication of the microfluidics chip as shown in Fig. 2a. An optical microscope photograph magnifying the electrode region is shown in Fig. 2b. The $30 \mu \mathrm{m}$ wide and $10 \mu \mathrm{m}$ high microchannels are fabricated to be the optimum channel dimensions that provide the highest sensitivity as well as being large enough for particles that prevent rapid clogging.

\subsection{Measurement and simulation}

The microfluidics chip was connected to a lock in amplifier (HF2IS, Zurich Instruments) via RG79 coaxial cables through a custom made probe station. The driver software for the lock in amplifier enables both real-time monitoring and data storage for further processing. Fluidic connections were achieved using silicon tubing with a metal syringe tip on the end. Three and $6 \mu \mathrm{m}$ diameter monodisperse polystyrene (PS) beads, which were procured from Poly Bead, were dispersed in the PBS solution. The solution conductivity was set to $2.5 \mathrm{~S} / \mathrm{m}$ using a conductance meter (Hannah).

FEM simulations were done using COMSOL 5.0 at the AC/DC module with a free tetrahedral mesh. Different type mesh elements were tested; however, the convergence of the solution cannot be provided with either triangular or quad meshing. COMSOL accepts two inputs in the material section, which were electrical conductivity and the relative 


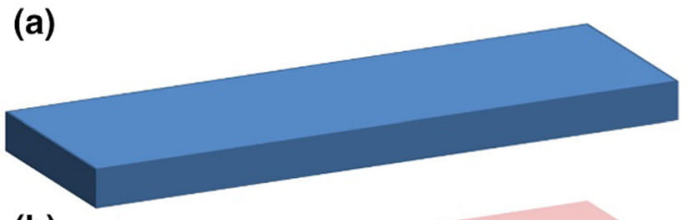

(b)

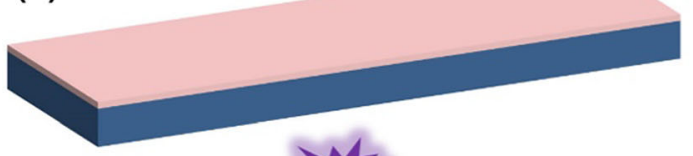

(c)

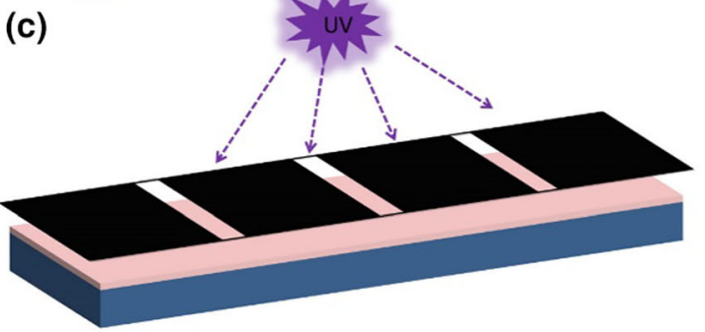

Fig. 1 Schematic representation of the micro electrode fabrication process. a Cleaning, b PR spin coating, $\mathbf{c}$ UV exposure through mask, d UV exposed PR become soluble, e By dipping into the developer, (d)

\section{(e)}
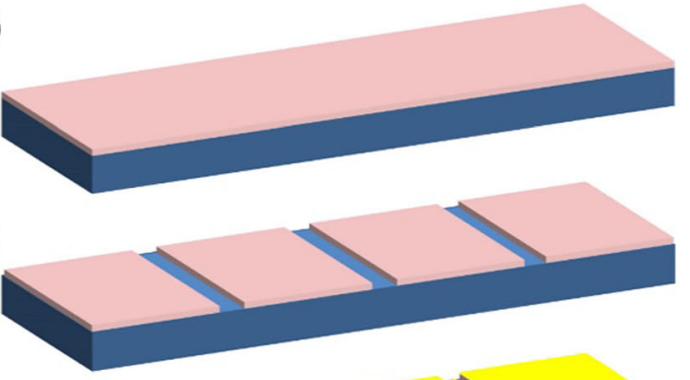

(f)

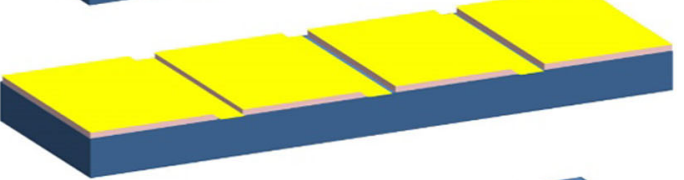

(g)

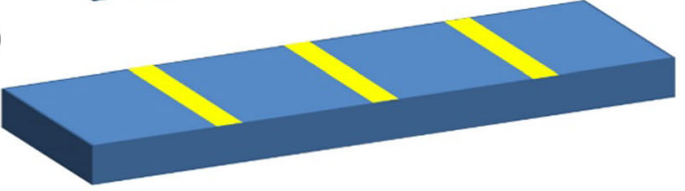

the exposed PR are removed, $\mathbf{f}$ Chromium and gold are coated with thermal evaporation, $\mathbf{g}$ Acetone etches only the PR leaving the metal over the glass surface
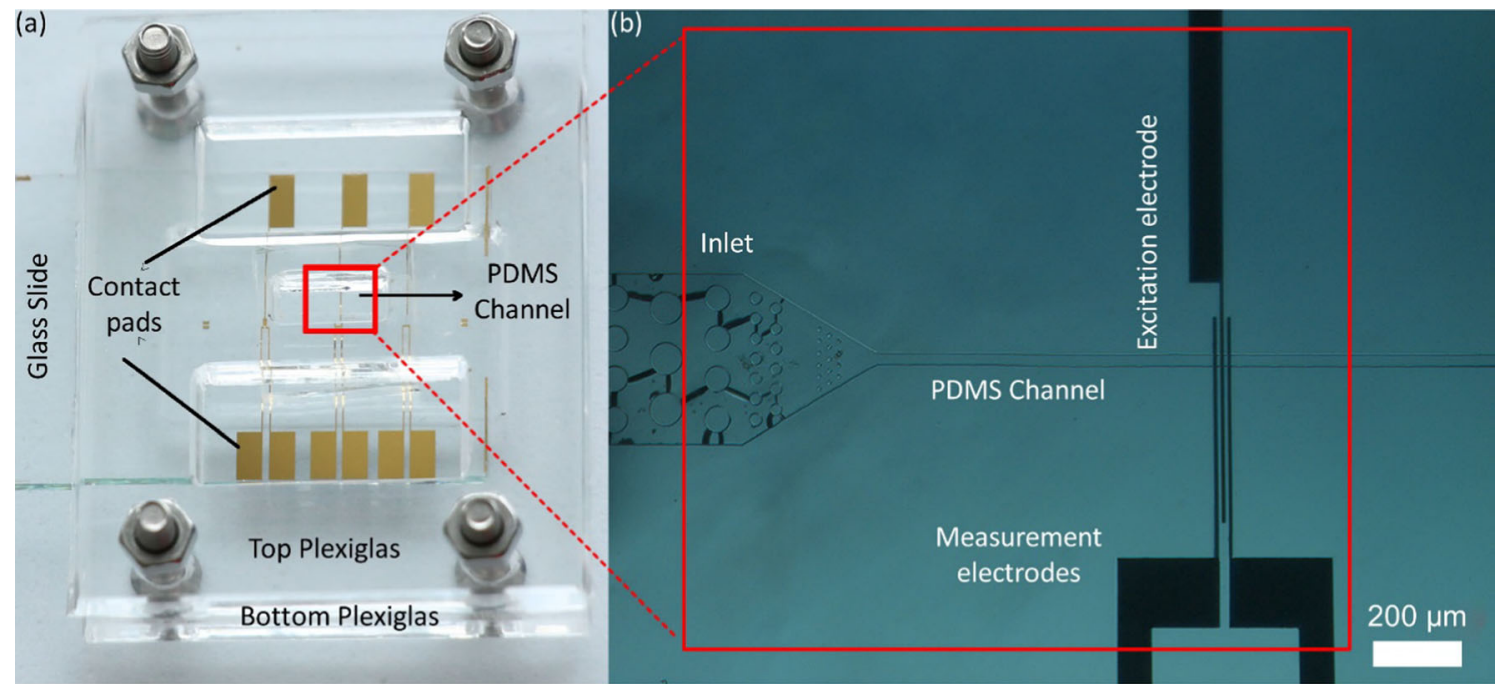

Fig. 2 a Microfluidics chip held by the laser-cut Plexiglas using nuts and bolts, b An optical microscope photograph of the microfluidics chip. Here, the PDMS microchannel can be seen crossing over the coplanar electrodes printed on the glass slide

dielectric constant. Hence, the two materials in the simulation that are the solution, and the particles, have to be assigned to related electrical parameters. In the real-world, in addition to those, there are PDMS, glass slide and electrodes material. However, the boundary condition

$J . n=0$

is assigned to the channel walls, and the electrode regions are assigned as terminal and ground, which removes the necessity to assign material parameters to them. COMSOL solves the maxwell equations in stationary format; as the frequency is constant, it can be written in the following version:

$\mathrm{E}=E_{0} e^{\mathrm{iwt}}$

Whose time derivative is given as follows:

$\frac{d E}{d t}=i w E_{0}$

Therefore the following equation is solved by employing FEM along with the meshes:

$\nabla \cdot(-\sigma+i w \epsilon) \nabla V=0$ 
$-\nabla V=E$

$\nabla \cdot j=Q$

Here $\sigma$ and $\varepsilon$ denote electrical conductivity and electrical permittivity of the medium; $\mathrm{j}$ denotes electrical current density; $\mathrm{n}$ denotes surface normal; $\mathrm{w}$ is the angular frequency of the excitation signal; $\mathrm{E}, \mathrm{V}$, and $\mathrm{Q}$ denotes electrical field, voltage, and charge respectively.

A representation of the solid and imaginary particle methodology of the simulation is shown in Fig. 3. To simulate the particle passing through the electrodes, a sphere is assigned with the electrical parameters of the polystyrene beads and the particle is moved through a $1 \mu \mathrm{m}$ space. The simulation is then run, and the total current is calculated at each point of the sweep from the COMSOL derived values module. The sphere assigned with the electrical parameters of the PS will subsequently be described as a solid particle, which is illustrated in Fig. 3a, b. The peak point of the current calculation for the solid particle is taken from the signal, which is shown in Fig. 3c. For the calculation of the noise at that current, the sphere is assigned with the same electrical parameters as the medium and the simulation is run with the same points used for the solid particle. Parametric sweep mode of the COMSOL is employed to simulate the solid and imaginary particles along the channel as if they are flowing suspended in the liquid by assigning the same parameter to both particle's x-axis position and running the sweep for 40-50 times. The current is calculated following the simulation. The sphere assigned with the solution parameters is called the imaginary particle as shown in Fig. 3d, e. The total noise is defined as the difference between the highest peak and the lowest trough values at the current output for this imaginary particle Fig. 3f. In this study, the solid particle is assigned with $10^{-6} \mathrm{~S} / \mathrm{m}$ electrical conductivity and a relative dielectric constant of 2.55 that represents the real PS particle. The imaginary particle was assigned with $2.5 \mathrm{~S} / \mathrm{m}$ electrical conductivity and a relative dielectric constant of 80 , sharing the same parameters as the medium.

The physics behind the electrical measurement is basically explained by Maxwell mixture theory (Maxwell 1873). Two different electrical measurement principles are

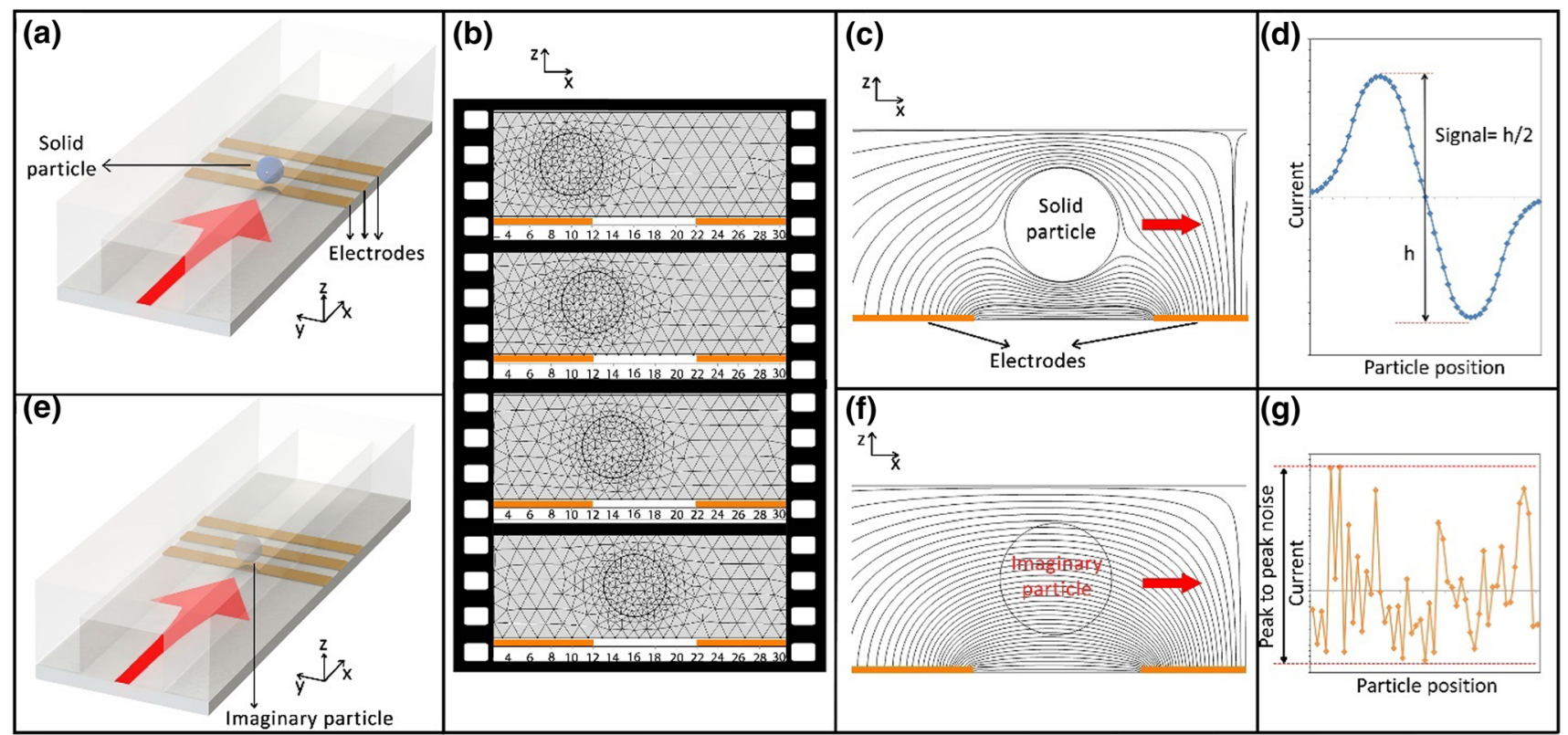

Fig. 3 Schematic representation of the particle in a microchannel simulated by COMSOL. Here the channel medium is assigned with the electrical parameters of the ionic solution. a The solid particle assigned with the electrical parameters of PS is simulated over the electrode region at points with $1 \mu \mathrm{m}$ spacing as if flowing through the channel, b Representation of mesh reordering due to particle simulated along the channel at different positions in 2D particle channel, and electrode system. For every new position of the particle, meshing changes to suit the emergent geometry c 2D simulation of the solid particle between the electrodes, which shows the blockage of the electric current lines by the solid particle, $\mathbf{d}$ The current variation generated by the solid particle is shown. Since the current coming from the first and the last electrode is subtracted, there is a peak and a trough. Here, the signal is taken as half of the change between the peak and trough and $\mathbf{e}$ The imaginary (ghost) particle assigned with the electrical parameters of the ionic solution was simulated over the electrode region as if flowing like a solid particle and $\mathbf{f} 2 \mathrm{D}$ simulation of the imaginary particle between the electrodes, which shows the electric current lines passing through the particle due to its same material properties with the medium, $\mathrm{g}$ The current variation generated by the imaginary particle is shown. Since there is no physically induced variation it is called simulation noise. Here the total noise is taken as the difference between the highest and the lowest points 
employed in this report, which are the double and the triple electrode methods. In the double electrode measurement, an $\mathrm{AC}$ voltage is applied to one of the electrodes that induces an electric current inside the channel. The current sensed at the opposite electrode goes to the current to voltage converter, in other words, a transimpedance amplifier (TIA). Here, the current signal is converted to a voltage through a $1 \mathrm{k} \Omega$ resistor and sent to the lock-in amplifier for signal processing that gives the root mean square (RMS) of the output. When a particle passes between the electrodes, the electric current is blocked due to the lower conductivity of the particle compared to the medium. The interrupted electrical current field lines lead to a reduction in the electric current prior to being transferred to the TIA, which is observed as voltage dips via the lock in amplifier. In the triple electrode design, excitation voltage is applied to the middle electrode and the current flowing from the outer electrodes is differentiated via an instrumentation amplifier after being converted to a voltage. Thus, a signal peak and trough is produced by a single particle passing over the electrodes due to subtraction of the signals. This process is done at the lock in amplifier by integrated components without any need for a custom made electronic setup. The frequency of the excitation voltage was determined to be $1.5 \mathrm{MHz}$ after an automatic frequency sweep to obtain the least noisy point. Here the noise is mainly thermal (Jhonson and Nyquist noise) as the frequency is relatively high preventing the Flicker noise (1/ $\mathrm{f}$ noise). However, analog to digital converter (ADC) components of the lock in amplifier having shot noise, quantization noise, and etc. contributes too. On the other hand voltage variations at the ground due to high power devices in the building as well as the open tip of the probes at the end of the coaxial cables, which are prone to the coupling of parasitic signals, brings the noise to the output. The measurements were sampled at $10 \mathrm{kHz}$ and analyzed with a custom-written Python code where pandas package was employed to handle the huge amount of data. For clarity, a small part of the measurement outputs are shown in the figure, but the average signal calculations are done over 500 peak points.

One can refer to a previous work demonstrating electrical current field lines and its relation to medium conductivity (Guler and Bilican 2018). Electrical flow cytometry is based on the observation of instant current variation, which is a sub branch of electrical impedance spectroscopy (Morgan et al. 2006). The technique is still in use in modern flow cytometers (Don 2003) and it is the foundation of all blood cell counters (Coulter 1953). Thanks to its highly sensitive nature, the electrical flow cytometry technique has many different applications, like analyzing stem cell differentiation (Song et al. 2013), leukemia cell detection from blood (Bilican et al. 2020b) or the analysis of engine lubricant oils (Murali et al. 2009). The sensitivity comes from the restriction of the electrical field lines thanks to microelectrodes and microchannels. The analysis can be done in an analytical domain by solving a boundary value problem for the Maxwell equations. However, the problem does not have an exact analytical solution for the situation where a particle is suspended between electrodes (Sun et al. 2007). The mathematics of nature makes it necessary to solve the problem in a numerical way.

The COMSOL simulations are done at the different mesh settings offered by the program itself. At the densest 3 levels there are finer, extra fine and extremely fine settings. All the parameters of the related mesh settings are given in Table $1 \mathrm{~S}$ and employed for all the related simulations. One should note that the mesh size can be set as low as required; these mesh settings are employed as offered by COMSOL, which is good enough for the purpose of this study. Currents flowing from the electrodes are calculated according to simulation outputs. The calculated current is multiplied by 1000 as if it is fed to lock-in amplifier through the TIA where it is converted to the voltage over a $1 \mathrm{k} \Omega$ resistor. As the lock-in amplifier gives the result by integrating the reference signal and the output signal over a period, RMS of the voltage value is calculated and plotted against the particle positions to enable a direct comparison of COMSOL and the lock-in amplifier output. The simulations are run on a desktop computer (HP workstation Z620, Intel Xeon E-5 2609 2.4 GHz CPU with $12 \mathrm{~GB}$ of RAM and a 64 bit Windows 10 operating system) at UNAM.

\section{Results and discussion}

The experimental measurements of the fabricated device were compared with the corresponding simulation results from the lock-in amplifier-which was invented to minimize noise during measurement and make them one of the most sensitive of all measurement devices-were compared with the FEM simulation. Lock-in amplifier results are smaller than the simulation results due to low pass filters that are employed to suppress the noise. Although being useful in reducing the noise, these low pass filters come with a disadvantage, which is the slow response such that when the particle is passing over the electrodes, low pass filters barely enables to increase the signal. In the meanwhile the particle leaves the electrodes and the signal disappears before reaching out to the real peak value. Thus, relatively lower peaks are observed via the lock-in amplifier then the calculated peaks due to truncation of the signal by low pass filters. 


\subsection{Double electrodes}

In Fig. 4a, the experimental output of the double electrode design is shown at a $1 \mathrm{~V}$ excitation signal. The peak to peak noise is $12.16 \mu \mathrm{V}$, while the average signal amplitude is $43.11 \mu \mathrm{V}$, which gives a signal to noise ratio (SNR) of 3.54. Here, the noise is mainly thermal (Jhonson and Nyquist noise) as the frequency is relatively high preventing the Flicker noise (1/f noise). However, analog to digital converter (ADC) components of the lock-in amplifier having shot noise, quantization noise, and etc. contributes to the noise in the output as well. On the other hand, voltage variations at the ground due to high power devices in the building, where the measurements are realized, as well as the open tip of the probes at the end of the coaxial cables, which are prone to the coupling of parasitic signals, bring the noise to the output too.

It was found that the mesh size is the most important parameter defining the intensity of the simulation noise. Here, the particle positions on the $\mathrm{x}$-axis are defined according to Fig. $4 \mathrm{c}$ for a simulation run in the parametric sweep mode during which COMSOL re-meshes the whole domain for each new point of the spherical object. One of the electrodes is assigned as the ground and the other one as the terminal at $1 \mathrm{~V}$. The AC signal frequency is set at $1.5 \mathrm{MHz}$, which is the same as the experimental frequency

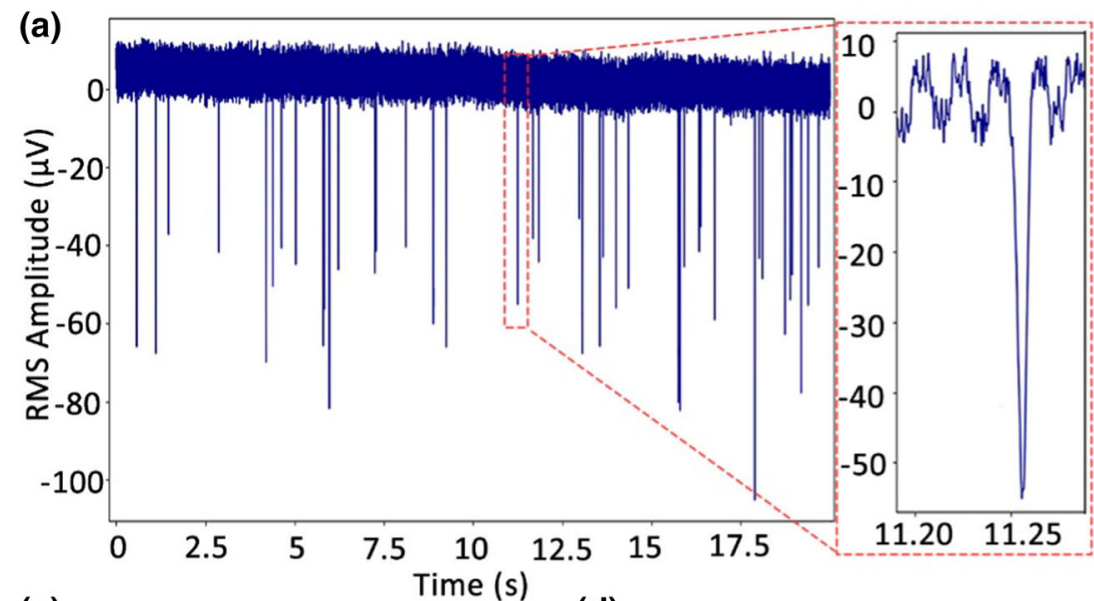

(c)

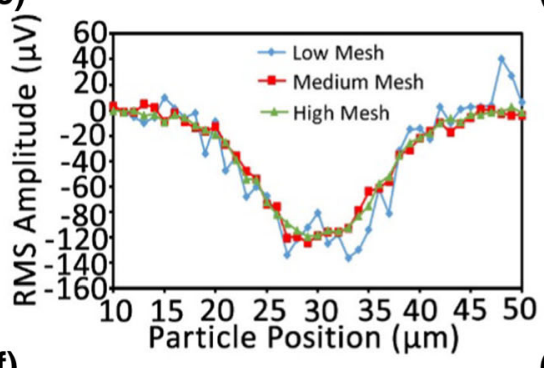

(f)

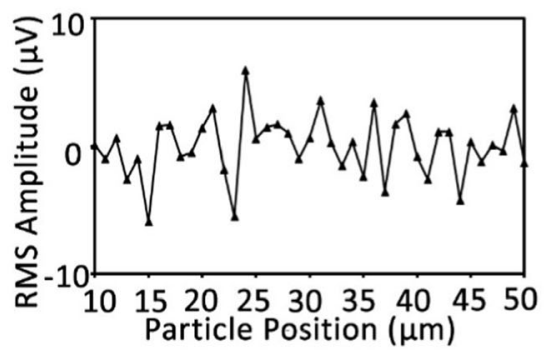

(d)

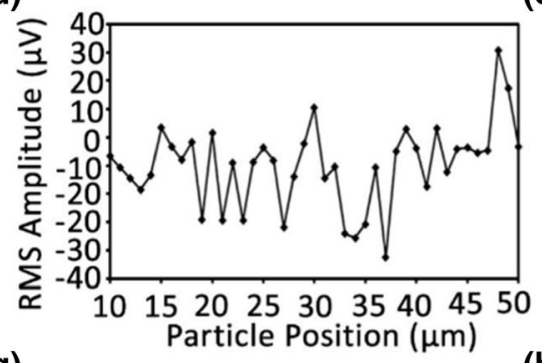

(g)

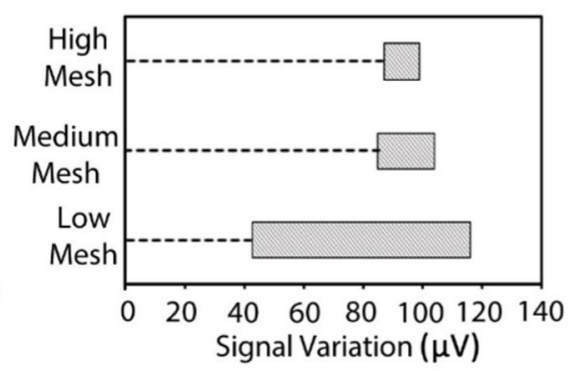

(e) (b)
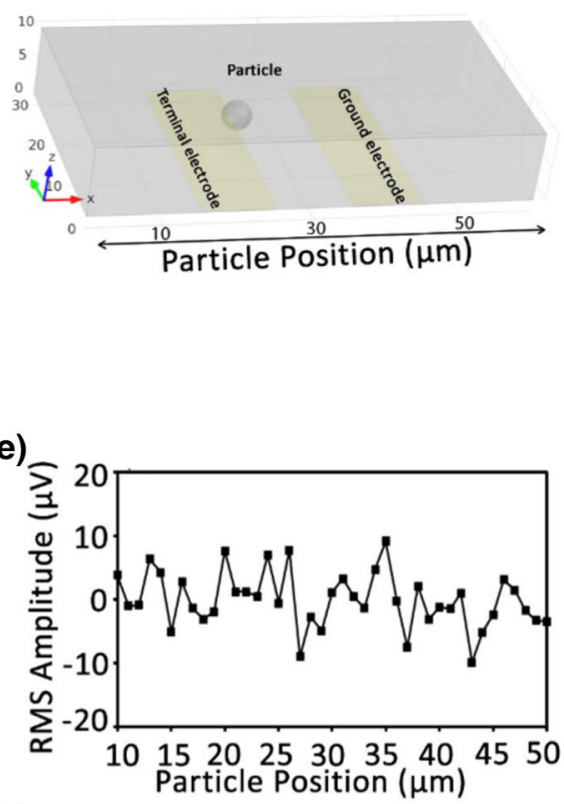

(h)

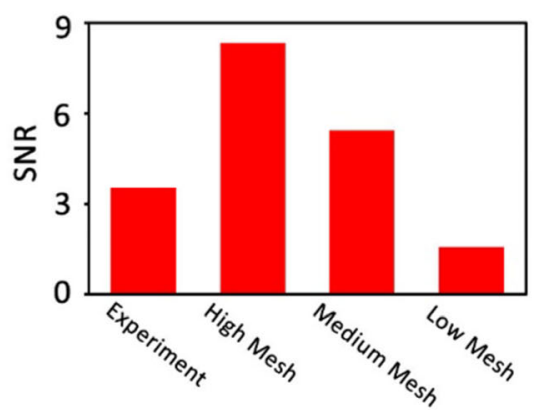

Fig. 4 Results of double electrode design for $3 \mu \mathrm{m}$ diameter particles. a Experimental output of PS particles; $\mathbf{b}$ COMSOL model, here the $\mathrm{x}$-axis demonstrates particle position along the channel where the particle (imaginary or real) is centered and simulated with $1 \mu \mathrm{m}$ spacing at 40 points. c The simulation results for the solid particles having the electrical parameters of PS at 3 different mesh settings Results of $\mathbf{d}$ The low density; e The medium density; f The high- density meshing for the imaginary particle where the imaginary particle have the electrical parameters of the medium which defines the noise at the simulation and shows a decreasing trend with rising mesh density. g Signal variation of the simulation output at different mesh settings $\mathbf{f}$ SNR values of experiment and simulations at different mesh settings 
set on the lock in amplifier. The simulation is run at each mesh setting for the solid and the imaginary particle. The height of the particle center above the base is set to $5 \mu \mathrm{m}$ to represent the average particle flowing in the real channel. According to the results, it gave a peak height of 116, 104, $99 \mu \mathrm{V}$ at low, medium and high mesh settings for the solid particle respectively, as shown in Fig. 4b. The imaginary particle gave 105, 37.5 and $18 \mathrm{nA}$ peak to peak noise levels as shown in $4 \mathrm{~d}-\mathrm{f}$ that produced SNR values of $1.58,5.45$ and 8.32, respectively, which is summarized in Fig. $4 \mathrm{~g}$.

The result proved that simulation has a noise source as well. The level of the noise decreased with increasing mesh density. While there is nothing to change the current level in terms of physics for the imaginary particle simulation, there is a strong fluctuation in the current level. Since the position of the particle changes the meshing-where the differential equations for the physical situation are solved - the current field lines are affected dramatically to yield a variation at the simulation output. Here, the experimental output had a better SNR than the low mesh settings but worse than the high mesh settings. However, neither the experimental nor simulation options for decreasing the noise are limited to the ones that have been described so far.

\subsection{Triple electrodes}

There are a few more ways to decrease the noise level experimentally, which have been tried one by one and compared with the related simulation results. One of the methods is to use the triple electrode design instead of the double electrodes. A $1 \mathrm{~V}$ excitation voltage is applied to the middle electrode (terminal 1) and $3 \mu \mathrm{m}$ diameter PS beads were sent through the channel. The electrical output measurement is shown in Fig. 5a where the average signal was $42.54 \mu \mathrm{V}$ with a $7.11 \mu \mathrm{V}$ peak to peak noise level giving an SNR value of 5.98. The COMSOL model of the microfluidics chip is shown in Fig. 5b. The solid particle gave a $141 \mathrm{nA}$ peak height as shown in Fig. $5 \mathrm{c}$ for all the mesh settings despite the noise level changes like those of the double electrode simulations. In this simulation, the current calculations were done by subtracting the current values of the outer electrodes, which was similar to the real experiment.

As shown in Fig. 5d-g, the noise level shows a descending trend with decreasing mesh size as expected. The peak to peak noise levels were nearly $96.4,33.9$ and $18.6 \mu \mathrm{V}$ at the low, medium and high settings, respectively. Hence, the SNR values were calculated to be $0.74,3.06$ and 5.39 at the related mesh settings from lower to higher density. According to the results, the triple electrode design improves the SNR value for only in the experiment not in the simulation. Here the experimental SNR is even better than the high mesh setting. Figure $5 \mathrm{~h}$ reveals the point where the same information-which is about the bead in the current case - can be obtained with more certainty using the triple electrode design in the experiment than the simulation.

\subsection{Particle size}

The effect of the particle size was investigated for both the experimental and simulation cases as another parameter. Figure $6 \mathrm{a}$ shows the experimental output for $6 \mu \mathrm{m}$ diameter PS beads where the peak to peak noise level was nearly $7.51 \mu \mathrm{V}$ and the average signal level was $262.31 \mu \mathrm{V}$ giving an SNR level of 34.88. Larger particles gave higher signals in accordance with the Maxwell Mixture Theory while the noise level was kept constant due to its dependence on the other experimental parameters. In the simulation as shown in Fig. $6 \mathrm{~b}$, the peak to peak noise was $101,41.1$ and $12.1 \mu \mathrm{V}$, which is nearly the same noise as the $3 \mu \mathrm{m}$ diameter particle as shown in Fig. 6e. It means that particle size doesn't make a significant change to the simulation noise. However, the signal level increases enormously due to the cubic growth of the particle volume with the diameter, see Fig. $6 \mathrm{c}$. Figure $6 \mathrm{~d}$ shows the signal variation for the simulation of the solid particle at different mesh settings, which was achieved by combining the imaginary and solid particle data. Results showed SNR values of 8.49, 19.96, 66.78 for $6 \mu \mathrm{m}$ diameter particles, see Fig. 6f. The experimental SNR was worse than the high mesh settings and better than the low and medium mesh settings of the simulation. It shows that the experiment and the simulation produced the same response to particle size in terms of noise, where the particle size does not affect the noise it only increases the signal.

\subsection{Excitation voltage}

Excitation voltage is the one applied to the middle electrode that induces a current between the other electrodes. Higher voltage means higher current which is sent to the lock in amplifier for processing. Thus, in the case of higher excitation voltage, the same size particle passing over the electrodes at the same position blocks more currents than the one running at a lower excitation voltage. Despite the same percentage change in the current in both cases, electronic circuits can handle higher voltage signals in a less noisy way. It is similar to the difference between being closer or nearer an FM radio transmitter, which affects the sound quality. Hence, at higher excitation voltages, the experiments produce higher SNR values, as shown in Fig. 7.

The experiments shown in Fig. $7 \mathrm{a}, \mathrm{b}$ are done at excitation voltages of $3 \mathrm{~V}$ and $0.5 \mathrm{~V}$ with $3 \mu \mathrm{m}$ diameter PS particles. Clearly, the SNR shown in Fig. 7a is much better than the SNR shown in Fig. 7b where the SNR at $3 \mathrm{~V}$ is 16.21 and the SNR at $0.5 \mathrm{~V}$ is 2.82 . Raising the excitation voltage enhances the SNR explicitly. However, the effect of the excitation voltage did not correspond to the simulation. Figure $7 \mathrm{c}, \mathrm{d}$ 


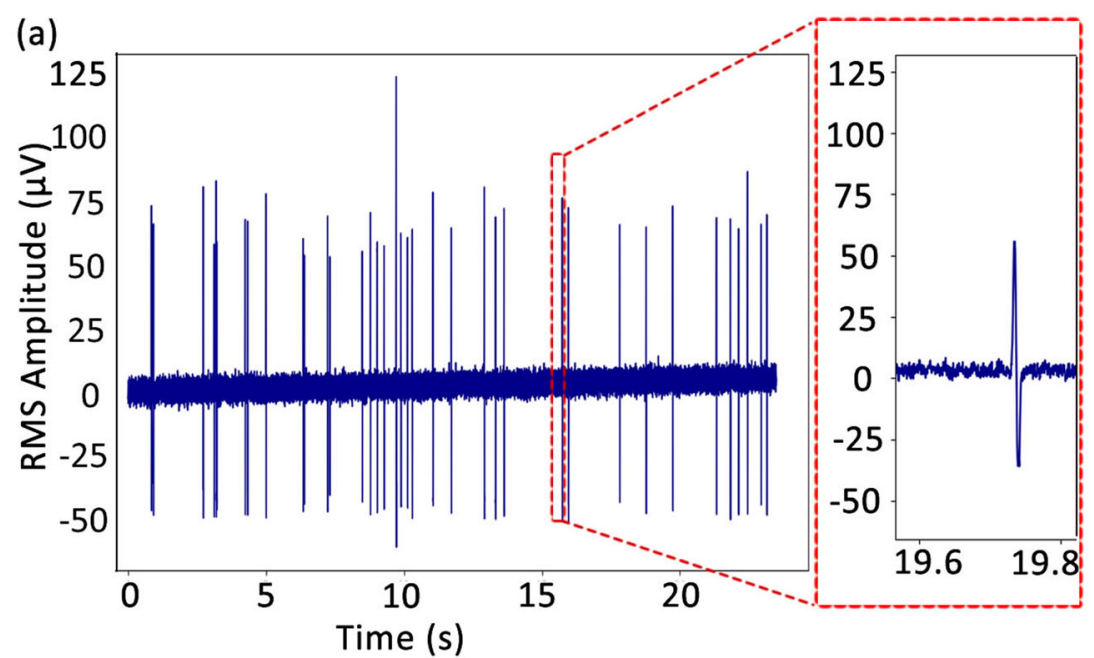

(c)

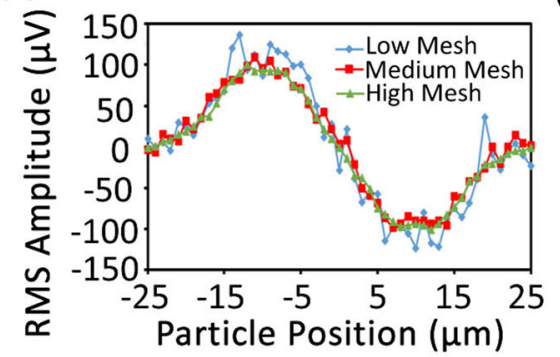

(d)

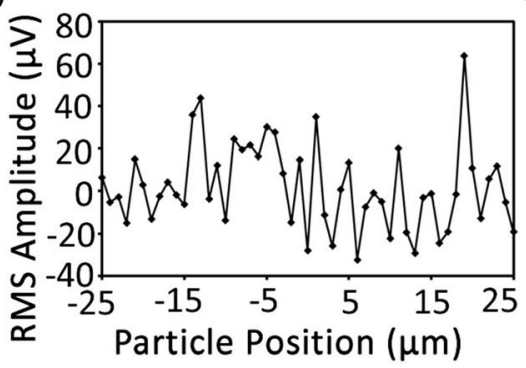

(b)

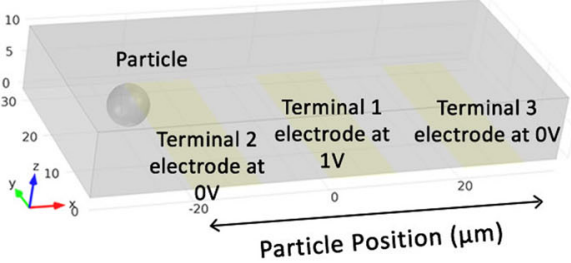

(e)

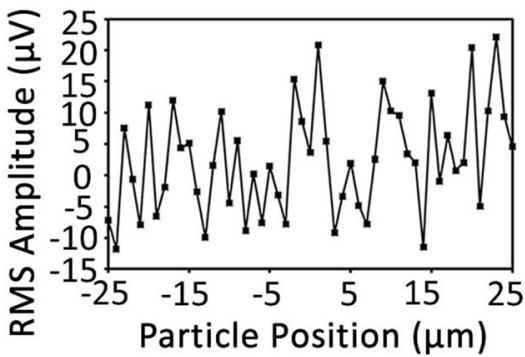

(f)

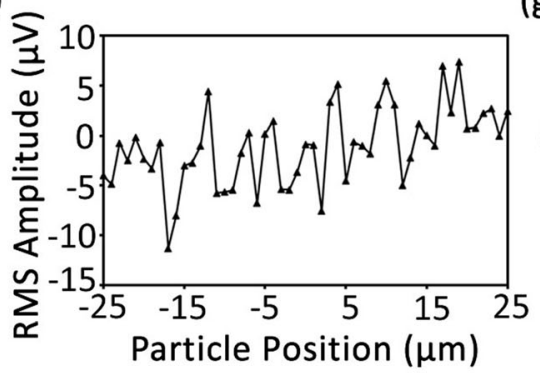

(g)

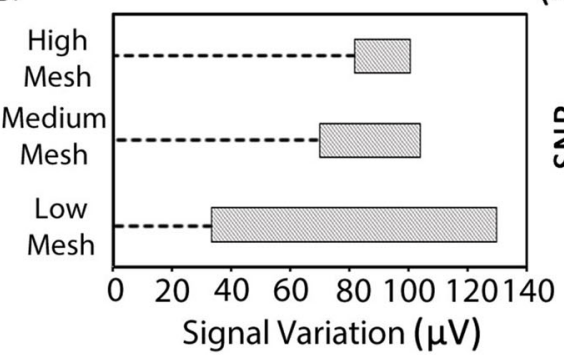

(h)

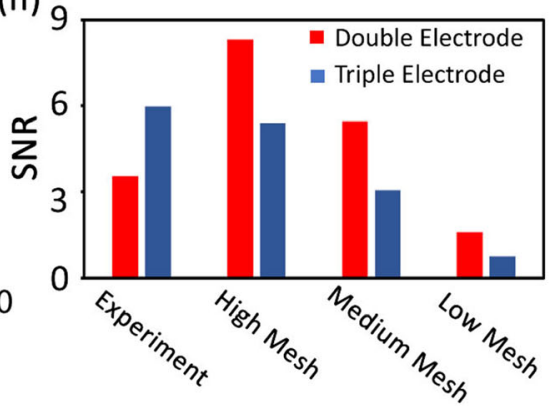

Fig. 5 Results with triple electrodes for $3 \mu \mathrm{m}$ diameter particles. a Experimental output of PS particles, b COMSOL model for the microfluidics chip The $\mathrm{x}$-axis shows the particle positions. Here the particle is centered at each point with $1 \mu \mathrm{m}$ spacing and the simulation is run for every new position of the particle each time, which is 50 repeat for the triple electrode design, $\mathbf{c}$ The simulation result of the solid particle assigned with the electrical parameters of

show the simulation of the same experiment at $3 \mathrm{~V}$ for the solid and imaginary particles, respectively, at the high mesh settings where the noise is $55.89 \mu \mathrm{V}$ and the signal is $302 \mu \mathrm{V}$, which produces an SNR of 5.39. Figure 7e, f show the same simulation done at an excitation voltage of $0.5 \mathrm{~V}$ where the signal is $50.25 \mu \mathrm{V}$ and the noise is $9.31 \mu \mathrm{V}$ to give an SNR of 5.39. Figure 7c, d, when compared with Fig. 7e, f-which is the $0.5 \mathrm{~V}$ version of the same simulation-shows that the noise increases 6 times matching the increasing excitation voltage, and explains the same SNR for both simulations. the PS at different meshing densities. The meshing noise achieved from the simulation results for the imaginary particle at, d low, e medium and $\mathbf{f}$ high density mesh settings. $\mathbf{g}$ signal variation of the simulation output at different mesh settings $\mathbf{h}$ Comparison of SNR levels of double and triple electrodes for experimental and simulation cases at different mesh densities

Since the voltage is just a boundary condition for the calculation, it affects both the solid and the imaginary particle simulation evenly. Therefore, Fig. $7 \mathrm{~g}$ shows that the excitation voltage does not affect the simulation SNR, whereas it affects the experiment dramatically.

\subsection{Particle height}

The effect of the particle height from the base of the channel was investigated as well. Since the electrodes are 
(a)

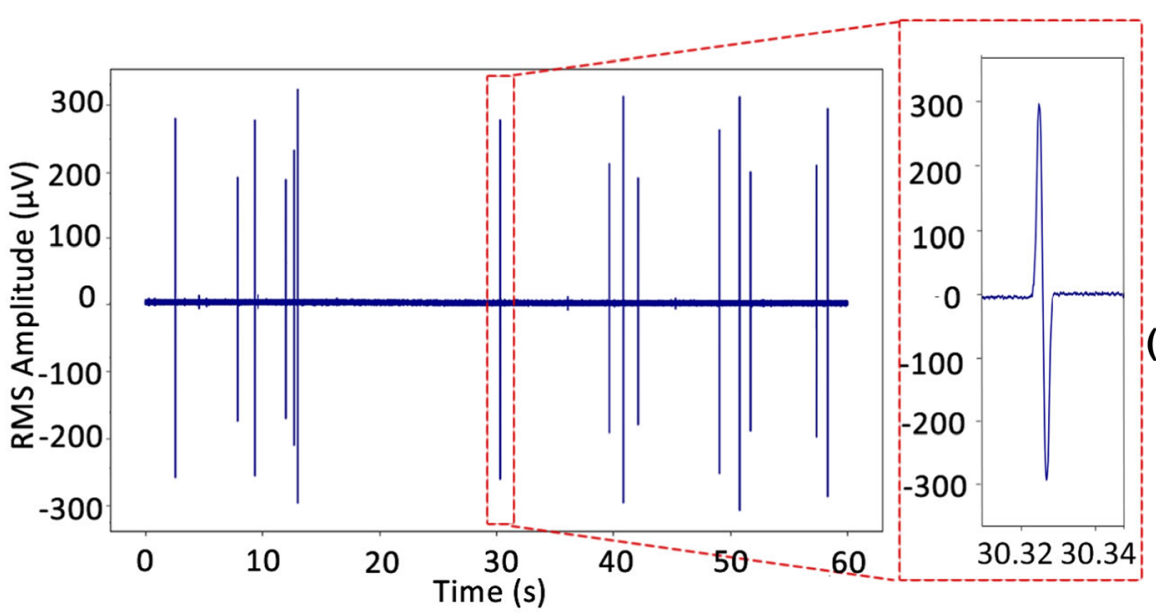

(e)

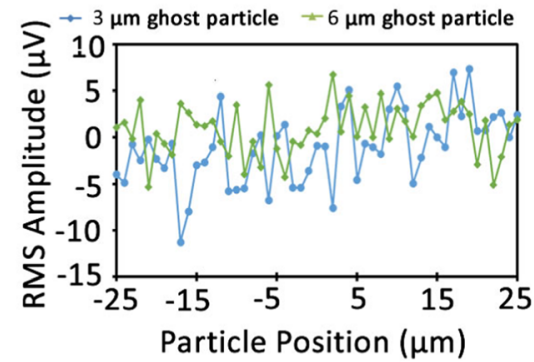

(b)

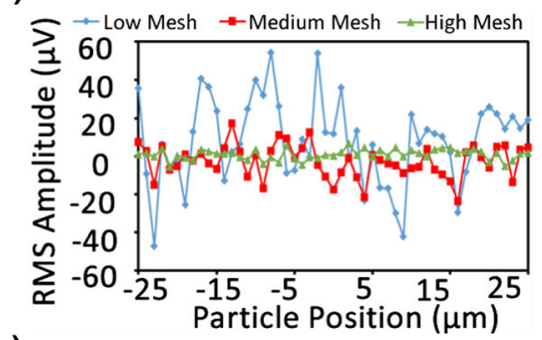

(c)

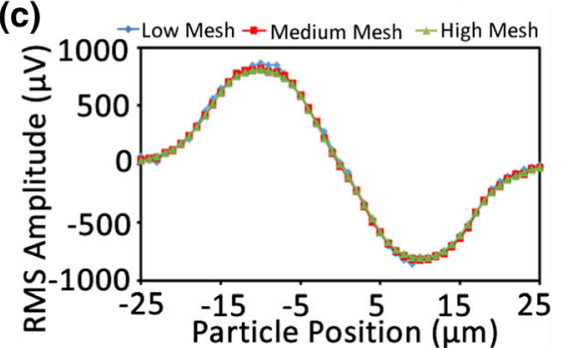

(d)

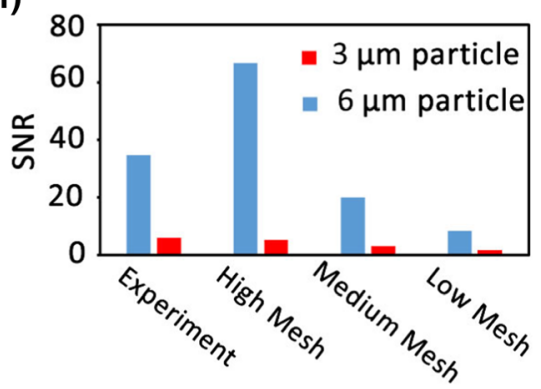

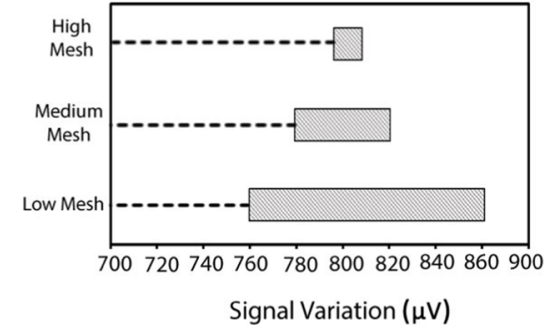

Fig. 6 The effect of the particle size on the experimental and simulation results a The measurement results for $6 \mu \mathrm{m}$ diameter particles, b The $6 \mu \mathrm{m}$ diameter imaginary particle at several meshing densities, c The $6 \mu \mathrm{m}$ diameter solid particle at several meshing densities and $\mathbf{d}$ signal variation of the simulation output at different

on the bottom, and the distance of the particle from the electrodes affects the results (Bilican et al. 2020a), the situation had to be investigated for the imaginary particles as well. Thus, a $3 \mu \mathrm{m}$ diameter particle was simulated at several heights from the bottom; the simulation results are shown in Fig. 8b-d. Here, Fig. 8a represents how the simulation is done based on the height, and which is defined on the figure itself. The standard deviations of the simulations are calculated for each height. According to the simulation outputs, the noise is dependent on the height. Figure $8 \mathrm{c}$ shows that the noise decreased moving away from the electrodes, which is reasonable due to the fact that the electric field is very strong near the electrodes, and distortion of the meshes near the electrodes induces more noise.

\subsection{Frequency}

The excitation frequency deserves to be talked about a little. It is well known that electrical impedance spectroscopy is highly frequency dependent (Guler and Bilican mesh settings e Comparison of the meshing noise produced by the 3 and $6 \mu \mathrm{m}$ diameter imaginary particle at high meshing. $f$ Comparison of SNR level for 3 and $6 \mu \mathrm{m}$ diameter particles for experiment and simulations of different density mesh settings

2018), which gives a better SNR at upper frequencies. However, the same behavior wasn't observed in the simulations. Since there is an electrical double layer in front of the electrodes behaving like a capacitor-which reduces its effectiveness at higher frequencies-simulations cannot represent the experiment at lower frequencies. Experimental results show that the SNR diminished very rapidly with decreasing frequency; however, it stayed constant from a few $\mathrm{Hz}$ to the $\mathrm{GHz}$ region in the simulation.

\subsection{Application to solid mechanics}

The method introduced here is not restricted to microfluidics impedance measurement, and it can be extended to other fields. To demonstrate that the idea is valid and applicable in different areas, a solid mechanics simulation was designed to calculate the deflection of a rectangular atomic force microscope (AFM) cantilever under a constant force. The designed cantilever has 2, 20, $200 \mu \mathrm{m}$ thickness, width, and height respectively having a conic tip at the end with $9 \mu \mathrm{m}$ radius and $20 \mu \mathrm{m}$ height. A typical 
(a)

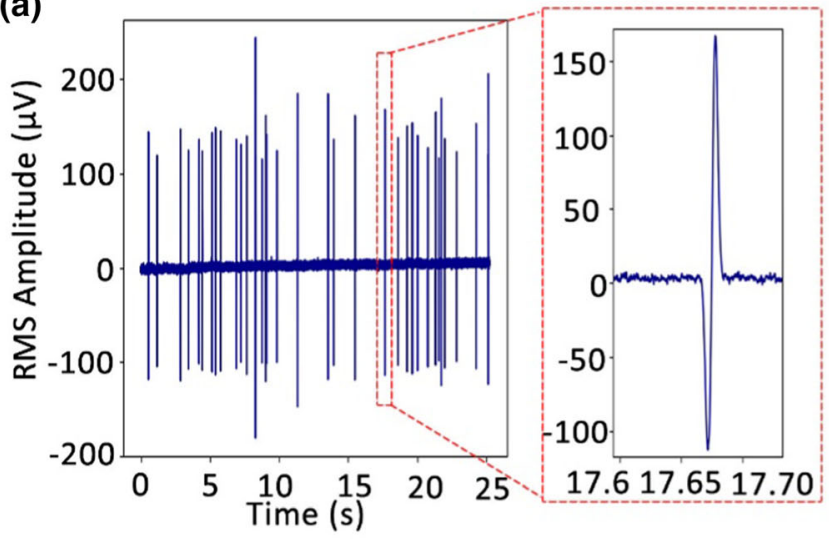

(c)

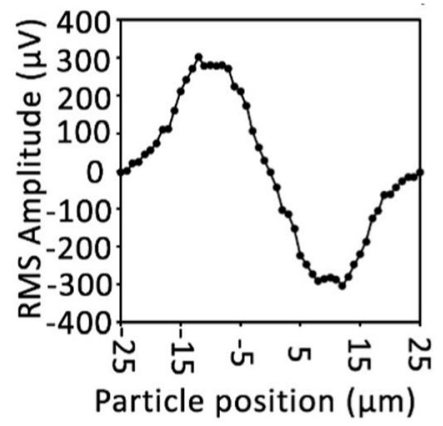

(d)

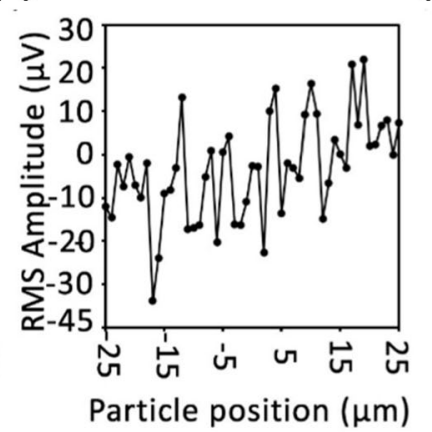

(b)
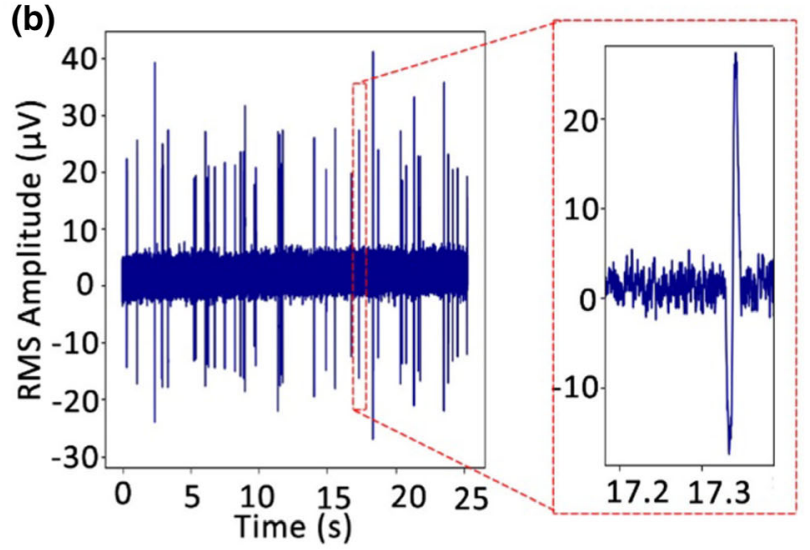

(e)

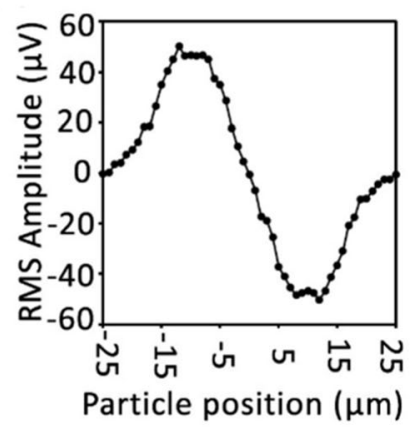

(f)

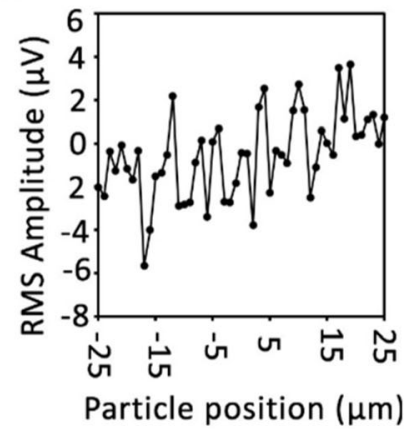

(g)

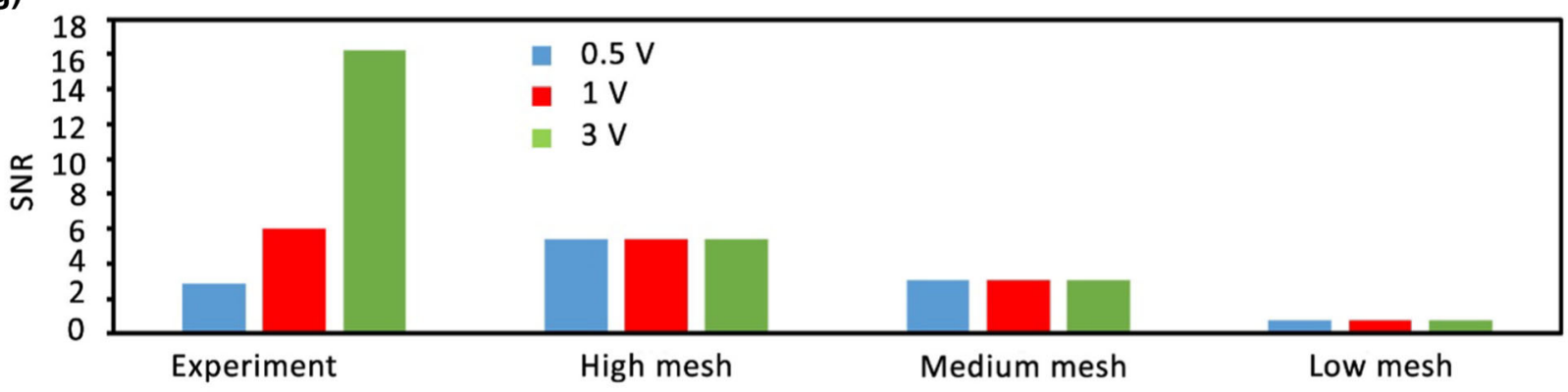

Fig. 7 Comparison of the effect of the excitation voltage on the noise for both the experiment and the simulation for $3 \mu \mathrm{m}$ diameter particles a Measurement of PS particles at $3 \mathrm{~V}$, b Measurement of PS particles at $0.5 \mathrm{~V}$, c Simulation of solid particles at $3 \mathrm{~V}$, d Meshing noise coming from the imaginary particle at $3 \mathrm{~V}$, e Simulation of

AFM force, $1 \mathrm{nN}$, is applied in the negative z-direction to the tip as well as a fixed constraint boundary condition applied to the beginning of the cantilever as shown in Fig. 9a where Silicon (100) is assigned to the entire geometry as the material. Spherical and prismal imaginary objects, as shown in Fig. 9d, assigned with Silicon (100) material properties, were moved along the cantilever in $\mathrm{x}$-direction beginning from $1 \mu \mathrm{m}$ till the end of the cantilever with $5 \mu \mathrm{m}$ steps.

The AFM analyses surface morphology by scanning the sample's surface with its tip that deflects up and down according to the applied effective force against the tip. The laser beam reflected from the cantilever surface is solid particles at $0.5 \mathrm{~V}$ and f Meshing noise achieved by the imaginary particle at $0.5 \mathrm{~V}$. g Comparison of SNR level for experiment and simulation at several excitation voltages, data shown for $1 \mathrm{~V}$ were drawn from Fig. 5

measured by a sensor that is correlated with the deflection magnitude. The AFM suffers from the thermal vibration of the cantilever that reduces the sensitivity and accuracy. A similar phenomenon is present in simulation which is demonstrated through imaginary particle method that put uncertainties on the results. Regarding the consistency of the previous SNR notation, the average value of total deflection is accepted as the signal amplitude and the absolute difference between the parametric sweep min and max values accepted as the noise as shown in Fig. 9b, c.

According to Fig. 9c, one thing that needs to be noted is that the bigger imaginary object produced slightly more noise. The other thing in that figure is the positional 
(a)

(b)

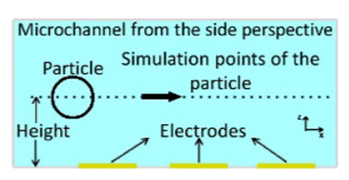

(c)

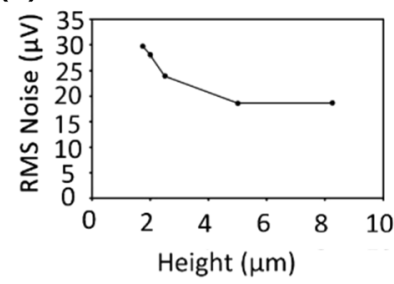

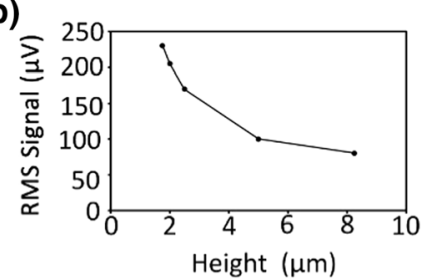

(d)

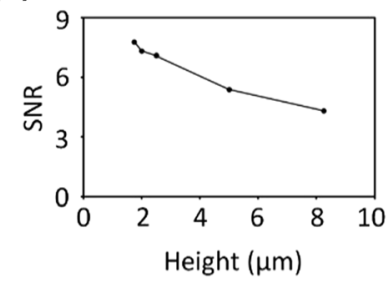

Fig. 8 Effect of the particle distance from the electrodes for $3 \mu \mathrm{m}$ diameter particles a Representation of the simulation. Here the height means the distance of the particle center from the surface where the electrodes are and $\mathbf{b}$ Signal achieved from the real particle at different heights $\mathbf{c}$ Standard deviation of the meshing noise from the imaginary particles of $3 \mu \mathrm{m}$ diameter at different heights $\mathbf{d}$ Height dependence of the SNR in simulation

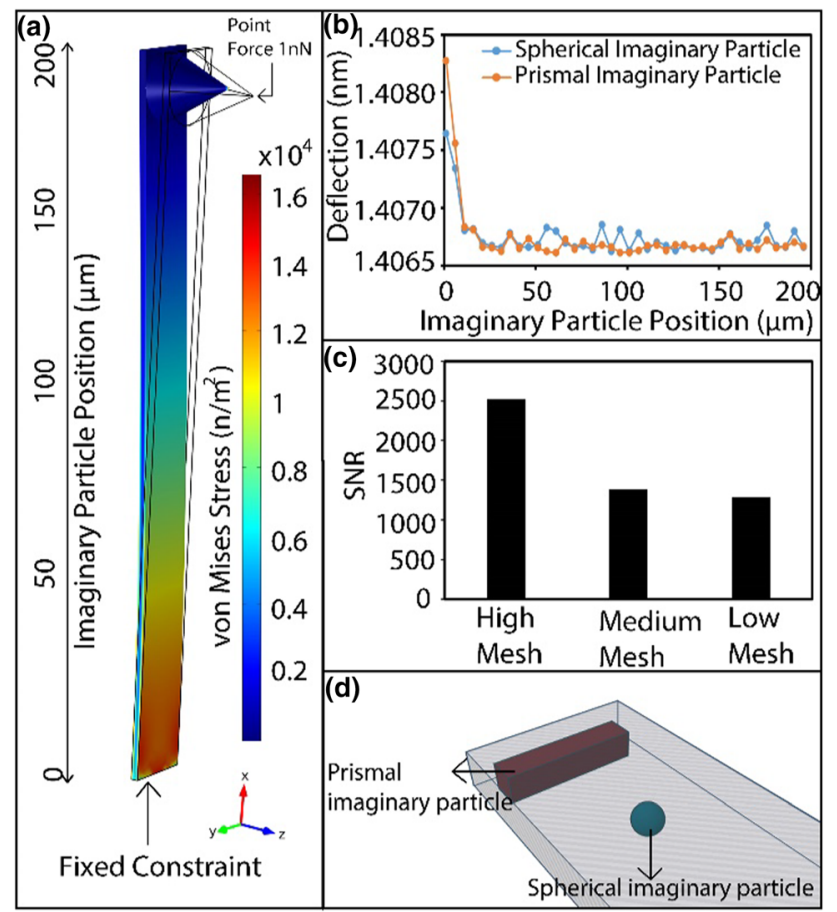

Fig. 9 Solid mechanics simulation. a The model shows a $200 \mu \mathrm{m}$ long AFM cantilver made of Silicon (100) where a point load is applied to the tip, and a fixed constraint boundary condition is applied to the other hand, $\mathbf{b}$ Comparison of the noise for the prismatic and the spherical imaginary object. Here the imaginary objects are moved from bottom to top with $5 \mu \mathrm{m}$ space and the simulation is re-run 40 times in total to give a higher noise level near the fixed constraint boundary, c Dependency of the SNR to the mesh density and d Illustration of spherical and prismal imaginary particles placed in the cantilever

dependence of the noise, which increases dramatically when the imaginary object moves closer to the fixed boundary region. Therefore, the noise-sensitive domains of the simulation model should be revealed by the imaginary object to truly specify the noise. The solid mechanics simulations showed that also the simulations based on physics other then electricity and magnetism can be done with an imaginary object sweep to determine the noise at the output, which was very significant in terms of assessing the limitations of the calculations. Therefore, uncertainties brought by the simulation noise to the theoretical calculations should be considered before carrying out an experiment, realizing a measurement, fabricating a device or designing a machine. In addition, Fig. 9c shows that the mesh density is the biggest constituent element in the meshing noise as expected, and whose simple parameters are introduced in Table $2 \mathrm{~S}$.

The simulation noise phenomenon is not limited to microsystems, it is also valid in the simulation of macro objects. A cantilever similar to AFM's cantilever, which is $10^{4}$ order of magnitude bigger, was designed and simulated to measure the deflection rate under a defined load. The cantilever having $1 \mathrm{~m}$ length, $20 \mathrm{~cm}$ width, and $1 \mathrm{~cm}$ thickness assigned with Aluminum alloy (6061) is loaded with $100 \mathrm{~N}$ from one side while keeping the other side fixed as shown in Fig. 1Sa. The mesh settings are given in Table $3 \mathrm{~S}$. The result shows that there is nearly 0.1 out of $57 \mathrm{~mm}$ uncertainty in the calculated deflection as given in Fig. 1Sb, c. The SNR shows mesh dependence character as well. This example demonstrates the applicability of this method to simulation from all scales.

\subsection{Computational cost versus noise}

The noise in the output defines a degree of uncertainty in the results for both the experiment and the simulation. In a simulation, CPU power and the time spent to complete the calculations are defined as the computational cost. The computational cost is proportional to computational complexity, which is the number of resources that an algorithm needs to finish the calculations. Time complexity, which means the amount of time to spend for a computer to solve the problem, is the most fundamental measure of the computational complexity. It is about the number of elementary operations of an algorithm to finish the calculation. Time complexity can be linear, quadratic, logarithmic, or another kind of a function of the input size. The most important parameter that a user can control in a package program like COMSOL is the mesh size. While there are some other parameters affecting the errors in FEM, like meshing quality or polynomial order (Kurowski 2017), only the mesh density was investigated in this study. According to the simulations done in triple electrode 
design with $3 \mu \mathrm{m}$ diameter imaginary particle, the computation time is linear with the mesh number, which means that the time complexity is linear against input size in accordance with the previous literature (Farmaga et al. 2011). Figure 10a shows that as the mesh number increases, the computation time increases linearly. There is a tradeoff between the mesh density and the noise, namely the uncertainty at the output. Similar to the Heisenberg uncertainty principle, one needs to sacrifice the time due to computational complexity in order to achieve more exact results. A plot indicating the correlation between computational cost in terms of time complexity and uncertainty is shown in Fig. 10b.

According to the trend line (red) shown in Fig. 10b, the following equation can be written:

$t^{3} n^{2}=C$

Here $\mathrm{t}$ defines the time to find the solution and $\mathrm{n}$ defines the noise at the result. Equation 7 means that the power of time to spend to find the result times the noise that brings uncertainty of the solution is constant.

In the real world there is no ideal sphere or a straight line; in theoretical studies assumptions are made, like

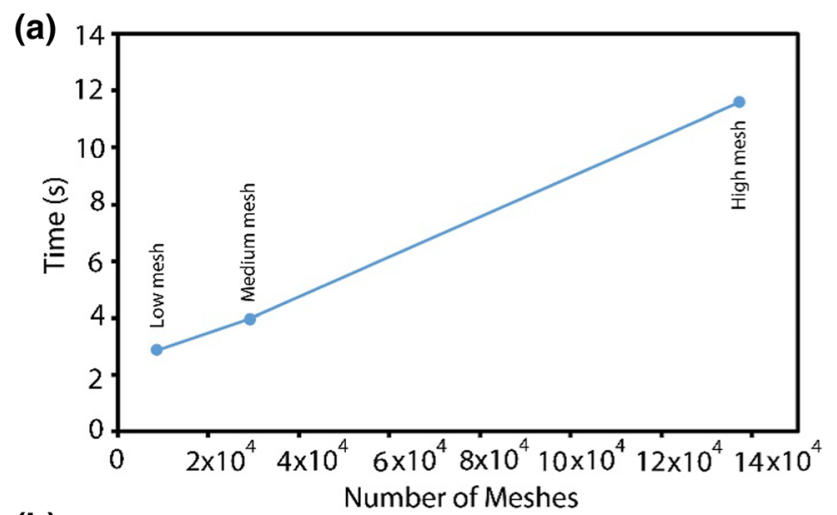

(b)

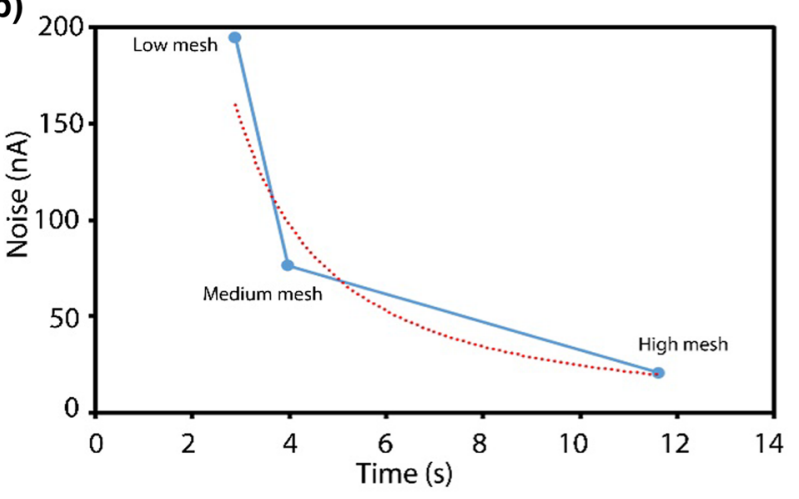

Fig. 10 a Computation time that corresponds to mesh number under low, medium, and high meshing densities is measured and plotted against the number of mesh. b Noise measured by the imaginary particle is plotted against computational time for (blue line). The red line defines the trend of the results taking the objects to be a perfect sphere or a straight line. In the case where no assumption or approximation is made, and considering all the parameters relevant to a problem, it is not possible to find an analytical solution for any physical problem. Hence, one needs to refer to numerical methods or do an experiment to analyze the physical situation. While only the FEM was investigated in the current study, the other numerical methods to solve a PDE have error sources as well. It shows that, like experiments in real life, the theory also features uncertainty because of the simulation noise, and the simulation is the only way to find the solution to a realistic PDE fully representing the physical case.

\section{Conclusion}

The definition of the imaginary object was that while it had no physical effect, it induced a fluctuation in meshing. Since the calculation of the partial differential equation governing the system was done through the meshes, outputs for the simulation produced variations as well. Hence, the meshing noise was defined as the output of the FEM simulation due to the imaginary particle. It was observed that the meshing noise reduced with increasing mesh density. Triple electrode sensing gave less noise than the double electrode sensing in the simulation and the experiment. Particle size didn't make a significant change to the simulation noise or the experiment, which demonstrated that the origin of the mesh variation may not be so effective. In some cases, the noise did not show such a corresponding trend in both the experiment and simulation. For example, the excitation voltage increased the SNR in the experiment, while it didn't make any improvement to the simulation results. The effect of distance from the most sensitive regions of the electrodes was also investigated. Results showed that the imaginary particles passing closer to the electrodes induced higher fluctuations, which meant increased meshing noise. Frequency also made no change to the meshing noise despite it dramatically affecting the noise in the experiment. However, it was mainly due to the electrical double layer which was not considered by the simulation. Despite the focus of this study being on AC electrical measurement in a microfluidics chip, the approach shown here shouldn't be restricted to AC electricity. It might be applied to all kinds of FEM simulations from fluid mechanics and solid mechanics to thermodynamics. Thus, another demonstration of the method was performed for a solid mechanics simulation that calculated the total elastic strain energy of a force applied to a material. No experiment was done to compare with these results; however, the method to measure the simulation noise worked well again. Therefore, by defining a 
imaginary object that does not have any physical effect that can be swept around the simulated system to remix the mesh points, fluctuation at the output can be achieved. The simulation results should be evaluated after determining the fluctuation dependent noise, and then the final design should be done according to the simulation results which include the specified uncertainty. By defining the uncertainty of the numerical results, any discrepancy between the simulation and the experiment should be removed, which will produce less faults and better designs. These findings show that due to the lack of an analytical solution for real problems in science and engineering numerical methods are the only way of analyzing some systems. Noise seems to be inevitable in all aspects of life, even theory.

Acknowledgements The author would like to thank to UNAM for the facility; Prof. Caglar Elbuken for critical reading of the manuscript; Dr. Ismail Bilican, Dr. Murat Serhatlioglu and Ali Kalantarifard for their kind helps in measurement setup, drawings and simulations.

\section{References}

Abidou D, Yusoff N, Nazri N, Awang MO, Hassan MA, Sarhan AA (2017) Numerical simulation of metal removal in laser drilling using symmetric smoothed particle hydrodynamics. Precis Eng 49:69-77. https://doi.org/10.1016/j.precisioneng.2017.01.012

Ames WF (2014) Numerical methods for partial differential equations. Academic Press, New York/London

Axelrad V (1998) Grid quality and its influence on accuracy and convergence in device simulation. IEEE Trans Comput Aided Des Integr Circuits Syst 17(2):149-157. https://doi.org/10.1109/ 43.681264

Axelrad V, Long G, Kuepper P (1997) Elimination of meshing noise in statistical TCAD. In: Paper presented at the 1997 2nd International Workshop on Statistical Metrology) doi: https:// doi.org/10.1109/IWSTM.1997.629425.

Azeman NSS, Nawi MNM, Yaacob MIH (2019) Development and characterization of fluidic based dome-shaped pressure sensor using spiral microchannel. Microsyst Technol. https://doi.org/10. 1007/s00542-019-04710-x

Banz L, Lamichhane BP, Stephan EP (2019) Higher order mixed FEM for the obstacle problem of the p-Laplace equation using biorthogonal systems. Comput Methods Appl Math 19(2):169-188. https://doi.org/10.1515/cmam-2018-0015

Bilican I, Guler MT, Gulener N, Yuksel M, Agan S (2016) Capacitive solvent sensing with interdigitated microelectrodes. Microsyst Technol 22(3):659-668. https://doi.org/10.1007/s00542-0152617-1

Bilican I, Bahadir T, Bilgin K, Guler MT (2020a) Alternative screening method for analyzing the water samples through an electrical microfluidics chip with classical microbiological assay comparison of $P$. aeruginosa. Talanta. https://doi.org/10.1016/j. talanta.2020.121293

Bilican I, Guler MT, Serhatlioglu M, Kirindi T, Elbuken C (2020b) Focusing-free impedimetric differentiation of red blood cells and leukemia cells: a system optimization. Sens Actuators B. https:// doi.org/10.1016/j.snb.2019.127531
Caire J-P, Chifflet H (2002) Meshing noise effect in design of experiments using computer experiments. Environmetrics 13(5-6):437-444. https://doi.org/10.1002/env.539

Caselli F, De Ninno A, Reale R, Businaro L, Bisegna P (2018) A novel wiring scheme for standard chips enabling high-accuracy impedance cytometry. Sens Actuators B 256:580-589. https:// doi.org/10.1016/j.snb.2017.10.113

Coulter WH (1953) Means for counting particles suspended in a fluid. Google Patents.

Dhara P, Singh VK (2020) Investigation of rectangular solid-core photonic crystal fiber as temperature sensor. Microsyst Technol. https://doi.org/10.1007/s00542-020-04927-1

Don M (2003) The Coulter principle: foundation of an industry. JALA-J Lab Autom 8(6):72-81. https://doi.org/10.1016/s15355535(03)00023-6

Errico V, De Ninno A, Bertani FR, Businaro L, Bisegna P, Caselli F (2017) Mitigating positional dependence in coplanar electrode Coulter-type microfluidic devices. Sens Actuators B 247:580-586. https://doi.org/10.1016/j.snb.2017.03.035

Farmaga I, Shmigelskyi P, Spiewak P, Ciupinski L (2011) Evaluation of computational complexity of finite element analysis. In: 2011 11th International Conference The Experience of Designing and Application of CAD Systems in Microelectronics (CADSM). IEEE, pp 213-214.

Gao Q, Zhang S (2019) Moving mesh method for simulating highdimensional time dependent PDEs with fast propagating shock waves. Eng Anal Boundary Elem 103:116-125. https://doi.org/ 10.1016/j.enganabound.2019.03.001

Gilden DL, Thornton T, Mallon MW (1995) 1/f noise in human cognition. Science 267(5205):1837-1839. https://doi.org/10. $1126 /$ science. 7892611

Guler MT, Bilican I (2018) Capacitive detection of single bacterium from drinking water with a detailed investigation of electrical flow cytometry. Sens Actuators, A 269:454-463. https://doi.org/ 10.1016/j.sna.2017.12.008

Hah D (2018) C-band optical filters with micromechanical tuning. Microsyst Technol 24(1):551-560. https://doi.org/10.1007/ s00542-017-3576-5

Handel PH 285 (1993) 'The nature of fundamental 1/f noise'. In: AIP Conference Proceedings. American Institute of Physics, pp.162-1711

Johnson JB (1928) Thermal agitation of electricity in conductors. Phys Rev 32(1):97. https://doi.org/10.1103/physrev.32.97

Kumar PA, Rao KS, Sravani KG (2019) Design and simulation of millimeter wave reconfigurable antenna using iterative meandered RF MEMS switch for 5G mobile communications. Microsyst Technol. https://doi.org/10.1007/s00542-019-04606-w

Kurowski P (2017) Finite element analysis for design engineers. SAE, Warrendale

Maxwell JC (1873) A treatise on electricity and magnetism. Clarendon Press, Oxford

Morgan H, Sun T, Holmes D, Gawad S, Green NG (2006) Single cell dielectric spectroscopy. J Phys D: Appl Phys 40(1):61. https:// doi.org/10.1088/0022-3727/40/1/S10

Murali S, Xia X, Jagtiani AV, Carletta J, Zhe J (2009) Capacitive Coulter counting: detection of metal wear particles in lubricant using a microfluidic device. Smart Mater Struct 18(3):037001. https://doi.org/10.1088/0964-1726/18/3/037001

Rahnama R (2020) Analyzing the piezoelectric rings resonance modes for bandwidth ultrasonic transducers. Microsyst Technol. https://doi.org/10.1007/s00542-019-04745-0

Reddy JN (1993) An introduction to the finite element method. McGraw-Hill, New York. ISBN: 9780070513556,0070513554

Saad A, Echchelh A, Hattabi M, El Ganaoui M (2018) An optimized control volume/finite element method (CV/FEM) for nonisothermal liquid composite molding (LCM) process. In: 
Thermo-mechanics applications and engineering technology. Springer, pp. 81-118. doi: https://doi.org/https://doi.org/10.1007/ 978-3-319-70957-4.

Serhatlioglu M, Asghari M, Tahsin Guler M, Elbuken C (2019) Impedance-based viscoelastic flow cytometry. Electrophoresis 40(6):906-913. https://doi.org/10.1002/elps.201800365

Shigyo N, Tanimoto H, Enda T (2000) Mesh related problems in device simulation: treatments of meshing noise and leakage current. Solid-State Electron 44(1):11-16. https://doi.org/10. 1016/S0038-1101(99)00220-8

Song H, Wang Y, Rosano JM, Prabhakarpandian B, Garson C, Pant K et al (2013) A microfluidic impedance flow cytometer for identification of differentiation state of stem cells. Lab Chip 13(12):2300-2310. https://doi.org/10.1016/S0038-1101(99)0022 $0-8$

Su T, Cheng K, Wang J, Xu Z, Dai W (2019) A fast design method for passive micromixer with angled bend. Microsyst Technol 25(11):4391-4397. https://doi.org/10.1007/s00542-019-04433-z

Sun T, Green NG, Gawad S, Morgan H (2007) Analytical electric field and sensitivity analysis for two microfluidic impedance cytometer designs. IET Nanobiotechnol 1(5):69-79. https://doi. org/10.1049/iet-nbt:20070019

Swati R, Wen L, Elahi H, Khan A, Shad S (2019) Extended finite element method (XFEM) analysis of fiber reinforced composites for prediction of micro-crack propagation and delaminations in progressive damage: a review. Microsyst Technol 25(3):747-763. https://doi.org/10.1007/s00542-018-4021-0

Thalluri LN, Guha K, Rao KS, Prasad GVH, Sravani KG, Sastry K et al (2020) Perforated serpentine membrane with AlN as dielectric material shunt capacitive RF MEMS switch fabrication and characterization. Microsyst Technol. https://doi.org/10.1007/ s00542-020-04755-3

Vo-Duy T, Truong-Thi T, Ho-Huu V, Nguyen-Thoi T (2018) Frequency optimization of laminated functionally graded carbon nanotube reinforced composite quadrilateral plates using smoothed FEM and evolution algorithm. J Compos Mater 52(14):1971-1986. https://doi.org/10.1177/0021998317737831

Voss RF, Clarke J (1975) '1/f noise'in music and speech. Nature 258(5533):317-318. https://doi.org/10.1038/258317a0

Wang FF, Shen JJ, Wu YY (2018) Passive tactile sensor for measuring elastic modulus of soft material: Continuum-mechanics model and experiment. Sens Actuators A 283:291-297. https://doi.org/10.1016/j.sna.2018.10.006

Wise ES, Cox BT, Treeby BE (2018) Bandwidth-based mesh adaptation in multiple dimensions. J Compu Phys 371:651-662. https://doi.org/10.1016/j.jcp.2018.06.009

Publisher's Note Springer Nature remains neutral with regard to jurisdictional claims in published maps and institutional affiliations. 